\title{
Diagnostic proteomics: Serum proteomic patterns for the detection of early stage
} cancers

\author{
Li-Rong Yu, Ming Zhou, Thomas P. Conrads and Timothy D. Veenstra* \\ Laboratory of Proteomics and Analytical Technologies, SAIC-Frederick Inc., National Cancer Institute at \\ Frederick, P.O. Box B, Frederick, MD 21702, USA \\ Tel.: +1 301846 7286; Fax: +1 301846 6037; E-mail: veenstra@ncifcrf.gov
}

\begin{abstract}
The ability to interrogate thousands of proteins found in complex biological samples using proteomic technologies has brought the hope of discovering novel disease-specific biomarkers. While most proteomic technologies used to discover diagnostic biomarkers are quite sophisticated, "proteomic pattern analysis" has emerged as a simple, yet potentially revolutionary, method for the early diagnosis of diseases. Utilizing this technology, hundreds of clinical samples can be analyzed per day and several preliminary studies suggest proteomic pattern analysis has the potential to be a novel, highly sensitive diagnostic tool for the early detection of cancer.
\end{abstract}

Keywords: Proteomic patterns, cancer detection, serum, mass spectrometry

\section{Introduction}

While having a tremendous impact on a variety of biological research areas, a major focus of proteomics is on the detection and identification of diagnostic biomarkers [10]. In proteomics, a biomarker is generally defined as an identified protein that is unique to a particular disease state. Experimentally, biomarkerdiscovery using proteomics strives to scrutinize clinical samples from healthy and afflicted individuals in a high throughput manner, allowing for the relative abundance of thousands of proteins from the two histopathologically distinct samples to be ascertained. Samples from healthy and diseased patients, for example, can be resolved and visualized on separate two-dimensional polyacrylamide gels. Protein spots that appear to be differentially abundant by staining techniques can be ex-

${ }^{*}$ Corresponding author: Dr. Timothy D. Veenstra, Biomedical Proteomics Program, SAIC-Frederick Inc., National Cancer Institute at Frederick, P.O. Box B, Frederick, MD 21702-1201, USA. Tel.: +1 3018467286 ; Fax: +1 3018466037 ; E-mail: veenstra@ncifcrf.gov. cised from the gel, digested, and identified using mass spectrometry (MS) [13]. The hope is that the identified protein(s) will indicate the pathological condition and therefore a diagnostic marker or therapeutic target for the disease of interest.

Despite the considerable intellectual and financial resources invested over the past decade in the use of conventional proteomic technologies for translational research, success in the discovery of novel diagnostic biomarkers has been remarkably poor. Reasons for the lack of success can be divided into two factors; technology-based and physiology-based. Technologically, the observed proteins are typically of high abundance and therefore valuable biomarkers that are expressed at low abundances are not routinely detected using current technology [23]. Comparison of just two samples from healthy and diseased patients using conventional proteomic technology is incredibly laborious and may not provide meaningful data. Furthermore, the identification of reliable and clinically useful biomarkers may require the comparison of thousands of samples. Physiologically, a useful biomarker would be ac- 


\section{Protein Arrays}

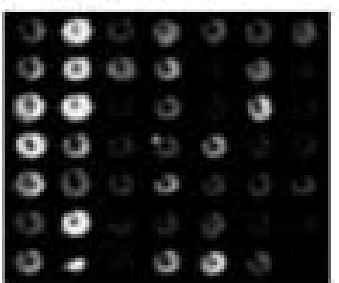

ICAT

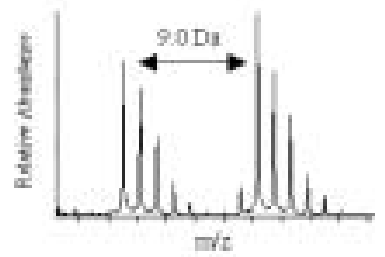

\section{D.PAGE}

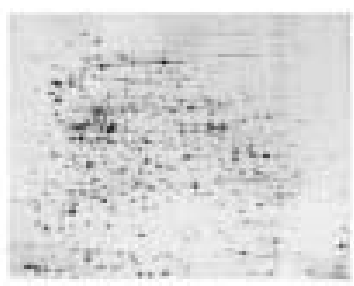

Proteomics in Biomarker Discovery

Mass Spectrometry Imaging

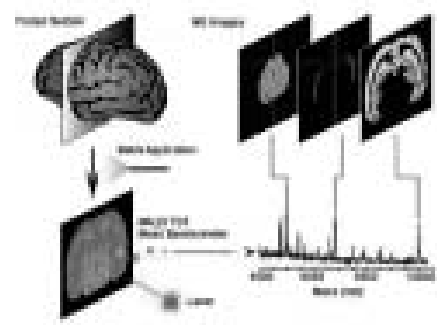

Mass Spectrometry

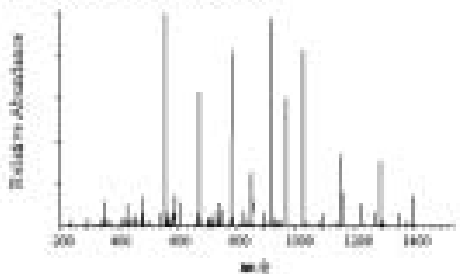

SELDI-TOF MS

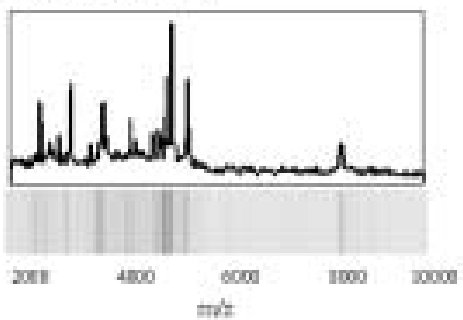

Fig. 1. A pictorial view of some of the various proteomic-based solutions used in the discovery of biomarkers.

cessible through an easily obtainable clinical sample such as serum, plasma, or urine. The natural variability of biofluids obtained from different patients makes the identification of a unique biomarker, within a sample with a constantly changing background matrix, quite challenging. In addition, a single, definitive biomarker for a particular physiological condition, such as human chorionic gonadotropin for pregnancy, may be quite rare [5]. Indeed, clinically accepted tests for such diseases as ovarian and prostate cancer through the detection of the biomarkers cancer-antigen (CA) 125 [9] and prostate specific antigen (PSA) [8], respectively, possess rather low positive predictive values (PPV).

Presently there exists several different proteomic based approaches that can be used to attempt to discover novel biomarkers. A few of these approaches are illustrated in Fig. 1. While mass spectrometry (MS) based approaches seem to dominate the search for biomarkers, there is a very active research program into the use of protein arrays that measure the abun- dance, or extent of modification of particular proteins through their interaction with specific affinity reagents such as antibodies or aptamers. Many of the MSbased approaches focus on the identification of differentially abundant proteins as indicated by their separation by two-dimensional polyacrylamide gel electrophoresis (2D-PAGE) followed by visualization by staining or by solution based differential analysis such as isotope-coded affinity tags (ICAT) in which proteins from different sources are labeled with isotopic variants of a cysteine-specific tag [25]. A novel approach to identify differences in protein abundances between cell types has been pioneered by Richard Caprioli. This approach, termed MS imaging, involves the direct analysis of tissue samples using matrix assisted laser desorption (MALDI) MS. In a recent application of this technique, MALDI-MS spectra were obtained directly from 1-mm regions of single frozen tissue of sections from 79 lung tumors and 14 normal lung tissues [24]. A class-prediction model was constructed using the 


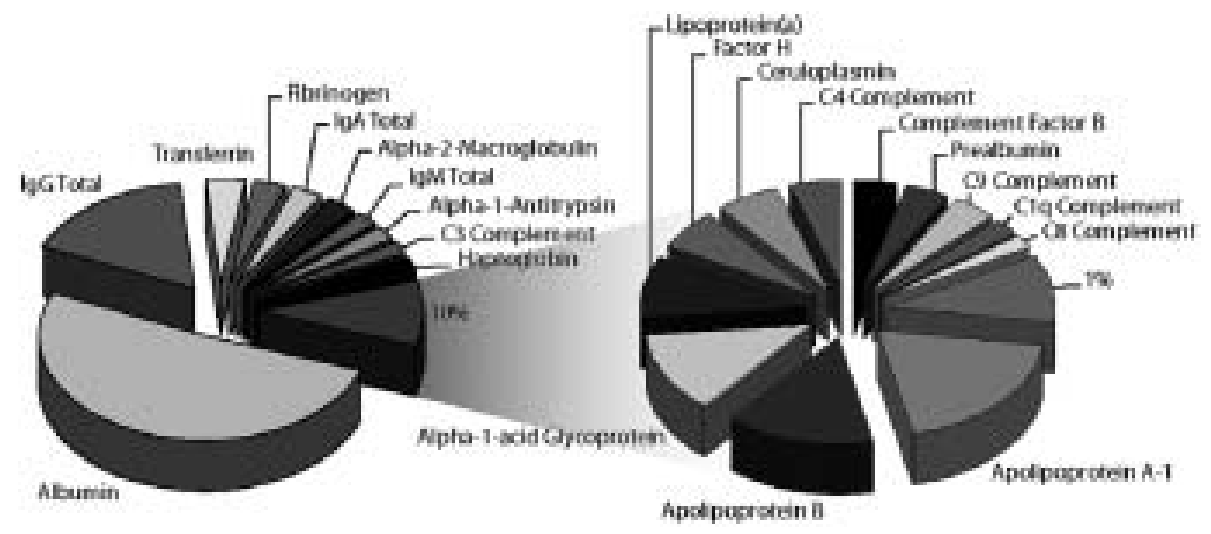

Fig. 2. Pie chart representing the relative contribution of proteins within plasma. Twenty two proteins constitute approximately $99 \%$ of the protein content of plasma.

proteomic patterns of a training cohort of 42 lung tumours and eight normal lung samples. This model was able to perfectly classify lung cancer histologies, distinguish primary tumours from metastases to the lung from other sites, and classify nodal involvement with $85 \%$ accuracy in the training cohort and nearly perfectly classified samples in the independent blinded test cohort. These results suggest that proteomic patterns obtained directly from small amounts of fresh frozen lung-tumour tissue can accurately classify and predict histological groups as well as nodal involvement and survival in resected non-small-cell lung cancer. One method that has attracted considerable attention over the past couple of years, however, has been surface enhanced laser desorption and ionization time-of-flight (SELDI-TOF) MS [12,19], which has contributed to the development of a potentially revolutionary method for diagnosing diseases through proteomic patterns, as describe below.

\section{Serum as a source of diagnostic information}

Serum is arguably the most important source of diagnostic information to describe the histopathological state of a patient. While serum potentially contains a plethora of diagnostic information, surprisingly little has been known about it protein content until very recently. Since serum has a very high protein concentration (i.e. $50-80 \mathrm{mg} / \mathrm{mL}$ ), this would make one think that serum is an ideal sample for proteomic analysis. Unfortunately, $99 \%$ of this protein concentration is made up by only 22 proteins, with albumin itself making up approximately $50 \%$ of serum's protein content (Fig. 2). The dynamic range of protein concentration has been estimated at approximately 9-10 orders of magnitude, making the characterization of the proteins within the lower $1 \%$ of protein abundance an analytically challenging endeavor.

Fortunately, as proteomic know-how and technologies have increased in their capabilities over the recent years, there are several studies that provide a glimpse into the protein makeup of this low abundance fraction of serum. One of the original studies used immunoglobulin (Ig)-depleted serum, which was digested with trypsin and fractionated using strong cation exchange chromatography (SCX) [2]. Each of the SCX fractions were characterized using microcapillary reversed-phase liquid chromatography coupled directly on-line with tandem MS ( $\mu$ LC-MS/MS). This study resulted in the identification of 490 unique proteins, the largest number of proteins identified in serum to date. While many of the highest abundant proteins, such as albumin, complement factors, etc. were identified, so to were several proteins, such as prostate specific antigen and interleukin-12, which are known to be present in very low concentration within serum. Another recently published study separated a tryptic digest of whole serum using preparative isoelectric focusing (IEF) prior to $\mu \mathrm{LC}-\mathrm{MS} / \mathrm{MS}$ peptide identification. In this study more than 300 serum proteins were identified [26]. Surveying both studies show that proteins from every biological and functional class were represented within serum, as well as proteins from every cellular locale. Taken together, these studies show that serum is made up of a wealth of proteins that are secreted or shed by cells that are either healthy or tumorigenic or dying. This characteristic of serum does make it an rich source of finding potential biomarkers for histopathological conditions. 


\section{Proteomic pattern technology}

Unfortunately, the inherent complexity of serum represents an overwhelming challenge to conventional proteomic approaches in which peptide abundances are compared and species of interest are subsequently identified. The comparison of just two serum samples using conventional proteomics technology will undoubtedly show many differences in protein abundances, however, determining the relevance of these changes to a specific disease state is very difficult. In addition, just comparing two serum samples in this manner would require days (if not weeks) of sample processing, data acquisition, and data processing time: much too slow and laborious for what needs to be done on a high-throughput basis. A revolutionary proteomic technology has recently been developed that does not rely on identification of any of the components within serum, rather it relies on the overall mass spectral pattern generated by a clinical sample of interest. The analytical methodology of the proteomic pattern approach is illustrated in Fig. 3. Proteomic patterns are acquired using surface enhanced laser desorption/ionization (SELDI) time-offlight (TOF) MS [12] as shown in Fig. 4. In SELDITOF MS raw biofluids are applied to a chromatographic surface of a protein chip that selectively retains components within the sample via adsorption, partition, electrostatic interaction, or affinity chromatography. One of the unique benefits, and what distinguishes SELDITOF MS, is that raw biofluids, such as urine, serum, and plasma, can be directly applied to the protein chip array surface. After a series of binding and washing steps, an energy-absorbing matrix is applied to each sample and a nitrogen laser is used to desorb and ionize bound species enabling their mass-to-charge $(\mathrm{m} / \mathrm{z})$ ratios to be measured by TOF MS. The result is a mass spectrum of the retained species on the protein chip array surface. The overall simplicity of the technology has contributed to the popularity of SELDI TOF MS to the biological community, as minimal expertise in the operation of MS instrumentation is required to generate mass spectral data.

\subsection{Application of proteomic patterns for disease diagnosis}

The potential of proteomic pattern analysis was first demonstrated in the diagnosis of ovarian cancer [19]. While ovarian cancer is not the most common cause of cancer-related deaths in women, $80 \%$ of women with common epithelial cancer are not diagnosed until the diseased is in advanced stages where the five-year survival rate is only $15-20 \%$ [18]. If diagnosed at stage 1 , the five-year survival rate rises dramatically to approximately $95 \%$ with surgical intervention. In this original study, the proteomic patterns of serum samples from several controls and ovarian cancer patients were acquired and a bioinformatic analysis, which combines elements of a genetic algorithm with cluster analysis [11, $15,16]$, was applied to the data to decipher diagnostic "patterns" within the profiles.

The analysis is divided into a pattern discovery and a pattern-matching phase. In the pattern discovery phase a set of randomly selected mass spectra of serum from healthy and ovarian cancer-affected individuals (i.e. the "training set") is analyzed to identify a subset of $m / z$ values and their amplitudes whose distinct presence distinguishes the normal serum spectra from the histopathological serum spectra. The bioinformatic searching process begins with hundreds of arbitrary choices of small sets (i.e. five to 20) of the $\mathrm{m} / \mathrm{z}$ values of the mass spectra. The diagnostic pattern is composed of the combined y-axis amplitudes of the candidate set of the key $m / z$ values. The pattern formed by the relative amplitudes of the chosen $m / z$ values is tested for its ability to distinguish the serum mass spectra acquired from the healthy and cancer-affected individuals. With the aim of identifying the pattern that provides the optimal segregation, the $m / z$ values within the most fit sets of features for distinguishing the training set spectra are reshuffled to form new feature sets and the resultant defined amplitude values are rated iteratively until the feature set that fully discriminates the preliminary sample spectra is revealed.

Once the most fit key $m / z$ values are selected, the diagnostic model, identified in the pattern discovery phase, is tested using masked spectra (i.e. the "testing set"). In this so-called pattern-matching phase only the key $m / z$ values and intensities in the feature set identified in the pattern discovery phase are used to classify the unknown samples as being from healthy or cancer-affected individuals. The diagnostic feature set defined in training was able to correctly diagnose the samples as being acquired from either control patients or those suffering from ovarian cancer with a sensitivity of $100 \%$ and a specificity of $95 \%$, yielding an overall PPV of 94\% [19]. The success in correctly deciphering stage I ovarian cancer suggested that proteomic patterns generated from biofluids may provide a useful indicator of the early onset of a particular disease state. 

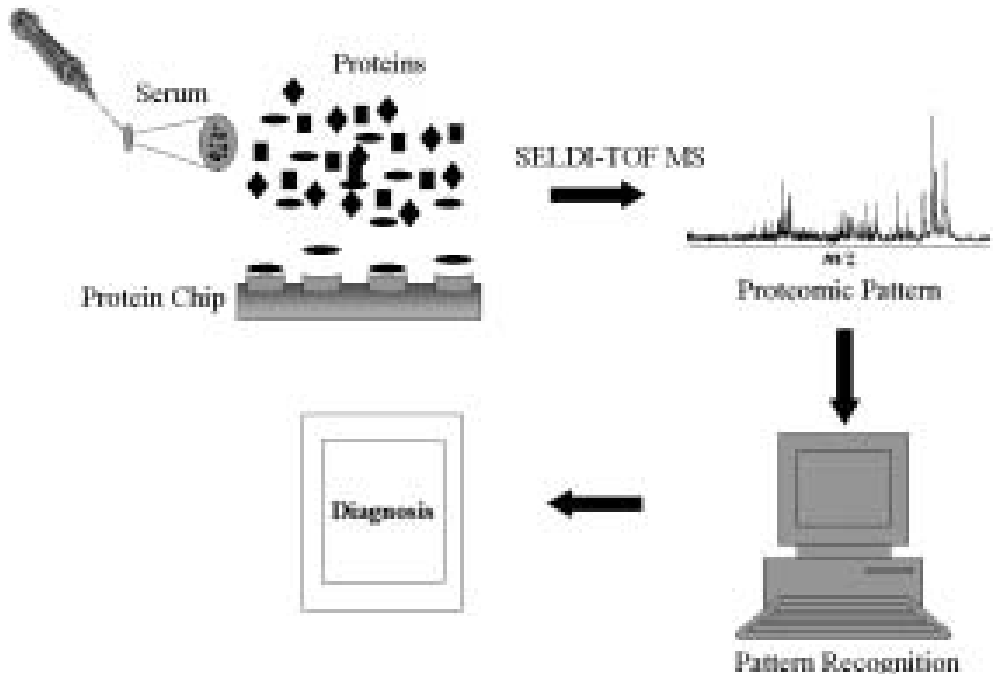

Fig. 3. Disease diagnostics using proteomic patterns. The serum sample is applied to a chromatographic surface that is arrayed onto a protein chip. After several washing steps, an energy-absorbing molecule is applied to the sample, and the mass spectrum of the species retained on the surface of the chip is acquired using laser desorption ionization (LDI) time-of-flight (TOF) mass spectrometry. The pattern of peaks within the spectrum is analyzed using sophisticated bioinformatic software to diagnose the source of the serum sample.

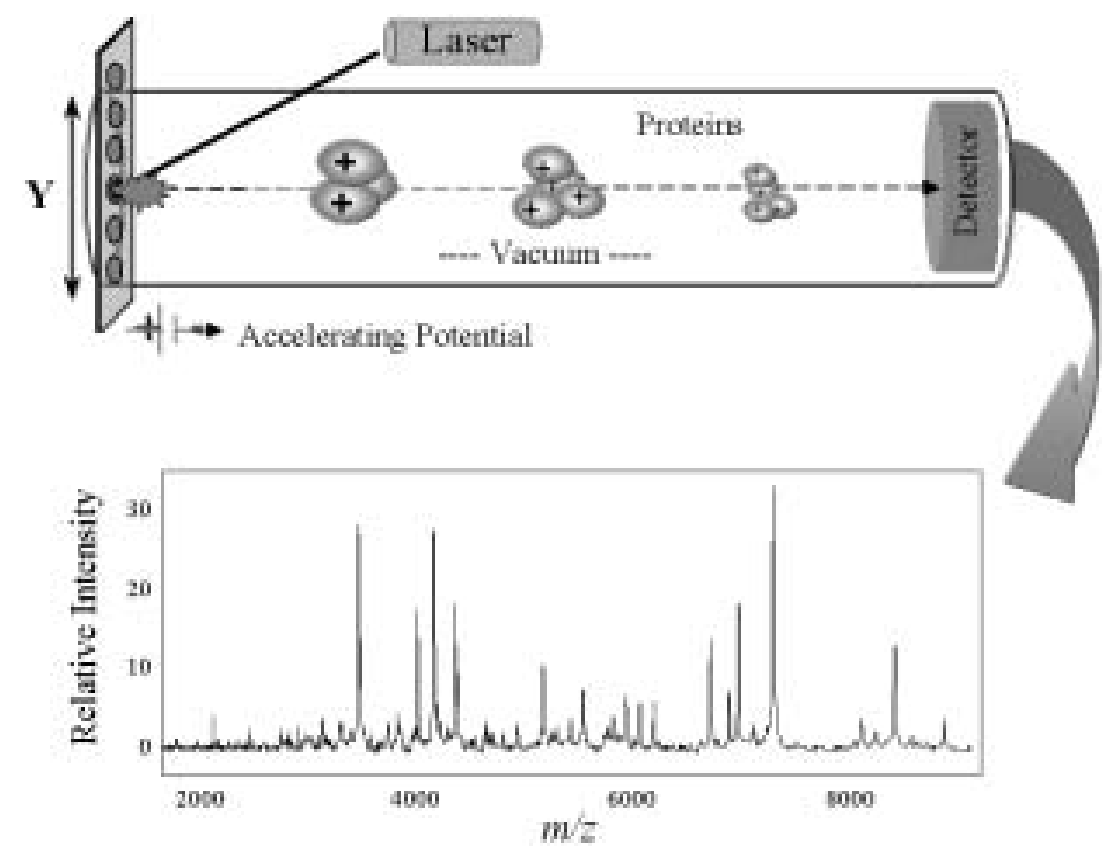

Fig. 4. Schematic diagram of the SELDI PBS-II mass spectrometer. After sample preparation the ProteinChip arrays are analyzed by a laser desorption ionization (LDI) time-of-flight mass spectrometer (TOF MS). The TOF MS measures the mass-to-charge $(\mathrm{m} / \mathrm{z}$ ) ratios weights of the various proteins that are retained on the array.

\subsection{Technologic comparison of three SELDI-TOF reports on prostate cancer}

While proteomic pattern analysis represents a potentially valuable method to diagnose early stage cancer, one major criticism of the technology is that the identity of the proteins or peptides giving rise to the key diagnostic features is not known [7]. As shown in Fig. 4, these features typically manifest as low intensity signals and developing methods to extract these potential 
biomarkers from a complex milieu such as serum is not trivial. At this stage in the development of proteomic pattern analysis it is debatable as to whether it is worth the effort to identify these features as they may provide little aid in developing an alternative diagnostic platform. Indeed, many of the diagnostic features are of low $m / z$ (i.e. $<10 \mathrm{kDa}$ ) and it is therefore likely that they are fragments generated from larger proteins that are proteolyzed in the tumor/host microenvironment. A recent study conducted in our laboratory to characterize components within the low molecular weight fraction of the serum proteome has shown that many of the identified peptides that pass through a $30 \mathrm{kDa}$ molecular cut-off membrane, originate from proteins with intact molecular masses much greater than the cut-off limit of the membrane [22]. It would be extremely challenging to generate an affinity reagent with specificity to a peptide fragment without considerable cross reactivity to its parent protein. Indeed, identification of a specific biomarker does not guarantee that this knowledge will provide any mechanistic or therapeutic insights into a particular cancer. A notable example of this situation is PSA. PSA is used to indicate the possible presence of a prostatic tumor, yet its role in cancer development remains unclear. Conversely, the identification of these diagnostic features is of considerable interest to the medical community and will likely be a major component of this technology in the near future.

Another major criticism is that studies using the same technology to develop a diagnostic for the same cancer, different peaks are recognized by the algorithms as crucial in distinguishing serum from healthy and diseased individuals. For example, three different prostate cancer detection studies reported $83 \%$ sensitivity at $97 \%$ specificity [1], $95 \%$ sensitivity at $78-83 \%$ specificity [20], and $97-100 \%$ sensitivity at $97-100 \%$ specificity [21]. The data from each study was roughly comparable and clearly superior to the specificity obtained by prostate-specific antigen (PSA) testing (25\%) while having similar sensitivities. It is surprising, however, that the two groups in three studies obtained these results using different methodologies and distinguishing peaks. Two of the groups used $[1,21]$ an IMAC-Cu metal-binding chip for serum adsorption while the third group used a hydrophobic C-16 chip [20]. While one group using the IMAC chips found that [1] nine peaks at $\mathrm{m} / \mathrm{z}$ ratios of $4475,5074,5382,7024,7820,8141$, 9149,9507 , and 9656 allowed serum from healthy and prostate cancer-afflicted individuals to be segregated, the study using the C-16 chips [20] selected different peaks at $\mathrm{m} / \mathrm{z}$ ratios of $2092,2367,2582,3080,4819$,
5439, and 18220. The other group that used IMAC chips [21] identified 12 major peaks at $\mathrm{m} / \mathrm{z}$ ratios of 9656, 9720, 6542, 6797, 6949, 7024, 8067, 8356, 3963, 4080, 7885, and 6991 for differentiating noncancer from cancer and 9 peaks at $\mathrm{m} / \mathrm{z}$ ratios of 7820,4580 , 7844, 4071, 7054, 5298, 3486, 6099, and 8943 for differentiating healthy individuals from patients with benign prostatic hyperplasia. There was no commonality between the peaks selected when comparing the results obtained using the IMAC and C-16 chips, however, more surprisingly, there were only two peaks in common (i.e. $m / z, 7024$ and 9656) when the studies performed using the IMAC chips were compared; even though these studies were performed using the same protein chips and mass spectrometer. One (albeit very unlikely) explanation to this discrepancy is fundamental to the analytical procedure while another is fundamental to the algorithm used to identify these key discriminatory features. As far as the analytical procedure, the spectral patterns acquired are very sensitive to experimental details such as how the serum is collected and stored. Any slight deviations from a standard protocol could result in changes in the proteomic pattern provided by a particular serum sample. As far as the algorithm is concerned, since serum is a very complex mixture of proteins and peptides there may be thousands of potential distinguishing peaks in serum. The chances that two different groups would find the same discriminating peaks using different instruments and computer algorithms would be extremely low.

\subsection{Instrumental improvements}

Several laboratories have subsequently shown the ability of serum proteomic patterns to diagnosis breast [17] and prostate [4,20,21] cancers with sensitivities and specificities greater than $90 \%$. While this diagnostic success rate is quite high, to function as an effective screening tool, a diagnostic assay screening for a low-prevalence disease such as ovarian cancer requires a specificity of at least $99.6 \%$ [14]. The need for such a high level of specificity for a clinical screening test can be rationalized if one considers that a false positive rate of even two percent for a low prevalence disease such as ovarian cancer would overwhelm the present medical system with unnecessary biopsies. Therefore, while proteomic pattern analysis in its present state represents a useful tool to confirm a diagnosis of cancer, its use as a screening tool for high-risk populations is still limited. 
All of the above mentioned studies have been performed using a PBS-II, which is a simple TOF-MS that is designed to provide for a broad $\mathrm{m} / \mathrm{z}$ detection range at the expense of resolution. A recent study compared the results of analyzing 248 serum samples from healthy and ovarian-cancer afflicted patients on a PBS-II and a hybrid quadrupole TOF (QqTOF) MS fitted with a SELDI ion source [6]. The resolution obtainable with the QqTOF MS is 60-fold higher than that obtainable with the PBS-II TOF MS, however, the spectra acquired on the different instruments are qualitatively similar.

Twenty-eight serum samples from unaffected women and 49 women with ovarian cancer were used for the training set in the bioinformatic analysis described above. A total of 108 diagnostic models were generated using a variety of different combinations of bioinformatic heuristic parameters. None of these parameters had any effect on the raw MS data, they were simply related to the bioinformatic process of generating diagnostic models from the raw data. These parameters included such things as the similarity space of likeness for cluster classification, the feature set size of random $\mathrm{m} / \mathrm{z}$ values whose combined intensities comprise each pattern, and the learning rate in training of the genetic algorithm. All of the models were derived and queried with the same set of proteomic pattern spectra. The models derived from the training sets acquired on the different MS platforms were tested using blinded serum sample mass spectra obtained from 31 unaffected women and 63 women with ovarian cancer. They were further validated using blinded serum sample spectra obtained from 37 unaffected women and 40 women with ovarian cancer. The diagnostic models generated from mass spectra acquired using the higher resolution Qq-TOF MS were statistically superior not only in testing but also in validation to those acquired on the PBS-II.

Quite importantly, four models were found that were both $100 \%$ sensitive and specific in their ability to correctly discriminate between serum samples originating from unaffected women and those suffering from ovarian cancer. Each of these models was generated with data acquired on the Qq-TOF MS, as no models with both $100 \%$ sensitivity and specificity could be found using the PBS-II data. No false positive or false negative classifications occurred using these models, giving each of these models a PPV of $100 \%$ using the patient cohort employed in this study. Each of these four models were able to correctly classify $22 / 22$ women with stage I ovarian cancer, 81/81 women with stage II, III and IV ovarian cancer, and 68/68 benign disease controls.
As opposed to the three prostate studies mentioned previously that gave differing key $\mathrm{m} / \mathrm{z}$ features within the most diagnostic models, the key $\mathrm{m} / \mathrm{z}$ features that comprise the four diagnostic models obtained using the QqTOF-MS data that had 100\% PPV for ovarian cancer revealed certain consistent features. Though the proteomic patterns generated from both healthy and cancer patients using the Qq-TOF MS are quite similar (Fig. 5), peaks at $m / z$ values 7060.121 and 8605.678 are more pronounced in a selection of the serum samples obtained from ovarian cancer patients as compared to unaffected individuals. This represented the first demonstration of consistency within diagnostic models and showed that several diagnostic models with high sensitivity and specificity can be obtained from a single set of data.

\section{Conclusions}

It is often anticipated that mass spectrometry will be used to identify the relevant biomarkers for a particular disease state and this information will be used to generate some type of affinity reagent (i.e. antibody, aptamer, etc.) that can be incorporated into an ELISAbased platform to screen serum samples for the presence of the biomarker originally identified by MS. The reasoning is that MS doesn't offer the throughput and reproducibility available using an ELISA-based system. There may be some technical difficulties associated with quantitating relevant serum biomarkers identified by MS. A recent study examining the low molecular weight proteome offers some clues to these difficulties [22]. In this study, serum was diluted fivefold in buffer containing $20 \%$ acetonitrile to disrupt non-covalent protein-protein interactions. The serum sample was then filtered through a $30 \mathrm{kDa}$ molecular weight cutoff membrane. The low molecular weight fraction that passed through the membrane was digested with trypsin and the resulting peptides analyzed by liquid chromatography coupled directly on-line with tandem MS so as to identify these peptides. Of the more than 800 unique peptides that were identified in this study, many were found to originate from proteins whose molecular weight was substantially greater than $30 \mathrm{kDa}$. One of these proteins, von Willebrand's factor has a molecular weight of $309 \mathrm{kDa}$. To confirm the presence of peptides from large proteins, the low molecular weight serum fraction was run on a SDSPAGE gel and several bands corresponding to molecular weights of less than $30 \mathrm{kDa}$ were excised from the 


\begin{tabular}{|c|c|c|c|}
\hline Model 1 & Mode1 2 & Model 3 & Model 4 \\
\hline 1276.861 & 818.450 & 1144.796 & 1001.654 \\
\hline 2374.244 & 6352.723 & 4260.403 & 1255.593 \\
\hline 4292.900 & 6548.771 & 7046.018 & 4377.854 \\
\hline 7060.121 & 7060.121 & 8602.237 & 6004.417 \\
\hline 8605,678 & 7096.922 & 8664.385 & 7060.121 \\
\hline 8706,065 & 8540.536 & & 7202.716 \\
\hline \multirow[t]{3}{*}{9870.938} & 8605.678 & & 8605.678 \\
\hline & 8706.065 & & 8709.548 \\
\hline & & & 9367.113 \\
\hline
\end{tabular}
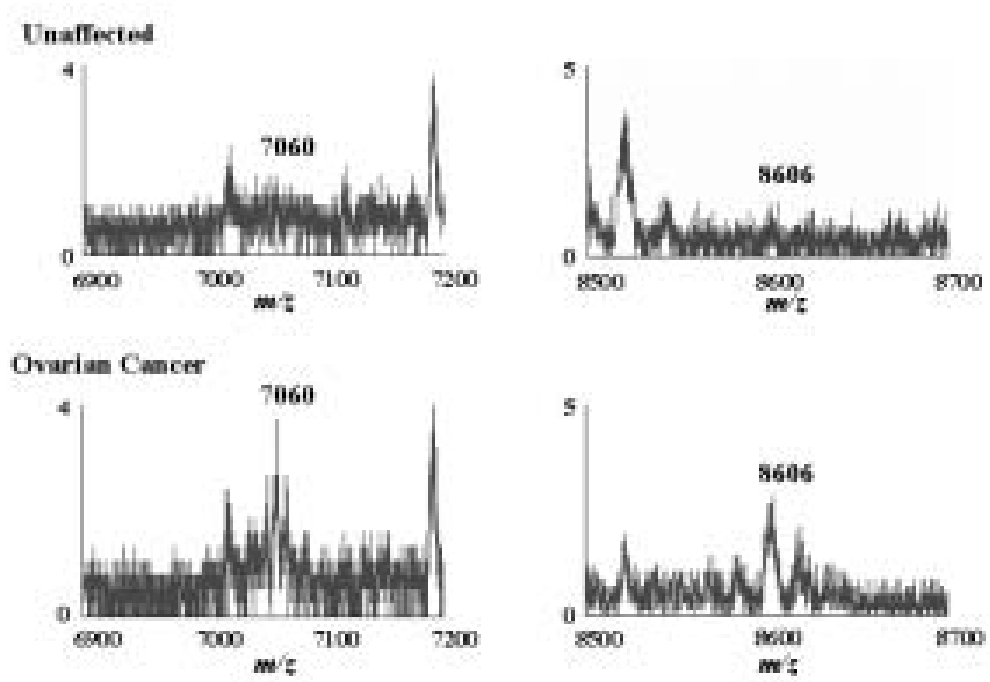

Fig. 5. Top: List of the key $\mathrm{m} / \mathrm{z}$ values in four diagnostic models that allowed serum acquired from healthy patients to be discriminated from serum acquired from ovarian cancer patients with $100 \%$ sensitivity and specificity. Bottom: Differential abundance of two key $m / z$ features selected in three of the four most diagnostic models differentiating serum acquired from unaffected and ovarian cancer-affected individuals.

gel. After performing and in-gel tryptic digestion, the peptides were extracted from the gel and identified by LC-MS/MS. Indeed several of these proteins that ran with an apparent molecular weight during SDS-PAGE less than $30 \mathrm{kDa}$, were identified as much larger proteins. These results suggest that many of the biomarkers identified as diagnostic for a particular disease state may be fragments from larger proteins. It would prove challenging to develop an affinity reagent that was specific for a fragment of a protein and did not cross-react with the intact protein itself. Probably the most important result to come from this serum characterization study was the finding that their exists an ocean of potential biomarkers within serum, as proteins from every general functional class, including oncogene products, were observed in this analysis.
One of the limitations of using individual cancer biomarkers is their lack of sensitivity and specificity when applied to large heterogeneous populations. It is likely that the measurement of a panel of biomarkers for a disease state can dramatically increase the overall diagnostic accuracy [3]. Biomarker pattern analysis is an emerging technology aimed at overcoming this limitation. While proteomic pattern analysis does not measure a panel of identified biomarkers, it does measure a panel of signals whose combination of $\mathrm{m} / \mathrm{z}$ values and relative amplitudes allows for the correct diagnosis. Focusing in on an individual peak, or subgroup of peaks does not provide the sensitivity and specificity attained from the combination of the diagnostic features. This multiplexed measurement makes inherent sense when one considers the systemic invasiveness of 
diseases such as cancer, in which the change of a single species due to tumor formation would be highly unlikely.

While disease diagnostics using proteomic patterns has rapidly emerged as a potentially revolutionary tool to detect and monitor disease progression or therapeutic response, it represents a complete about face in proteomic analysis. The major thrust in proteomics using MS-based technology over the past five years has been to identify and characterize an increasing number of proteins from a particular clinical sample in order to find a disease-specific biomarker. Diagnosing histopathological conditions via a proteomic pattern instead of assaying an identified disease-related biomarker represents a new paradigm in the use of MSbased tools for the discovery of diagnostic markers. The diagnosis of diseases, such as cancer, using proteomic patterns holds great promise. Since it is a relatively new concept, however, much of the entire process, including sample acquisition and processing, pattern acquisition, and data analysis requires optimization. The success of using this technology as a screening tool to detect early stage cancer, for example, will require recognition and establishment of strict quality controls so that samples being analyzed within different laboratories are treated identically. While many valid criticisms still abound, the high sensitivity and specificity that has been shown in several studies using proteomic pattern as a diagnostic test cannot be ignored.

\section{Acknowledgements}

This project has been funded in whole or in part with Federal funds from the National Cancer Institute, National Institutes of Health, under Contract No. NO1CO-12400.

By acceptance of this article, the publisher or recipient acknowledges the right of the U.S. Government to retain a nonexclusive, royalty-free license and to any copyright covering the article. The content of this publication does not necessarily reflect the views or policies of the Department of Health and Human Services, nor does mention of trade names, commercial products, or organizations imply endorsement by the US Government.

\section{References}

[1] B.L. Adam, Y. Qu, J.W. Davis, M.D. Ward, M.A. Clements, L.H. Cazares, O.J. Semmes, P.F. Schellhammer, Y. Yasui, Z. Feng and G.L. Wright Jr., Serum protein fingerprinting coupled with a pattern-matching algorithm distinguishes prostate cancer from benign prostate hyperplasia and healthy men, Cancer Res 62 (2002), 3609-3614.

[2] J.N. Adkins, S.M. Varnum, K.J. Auberry, R.J. Moore, N.H. Angell, R.D. Smith, D.L. Springer and J.G. Pounds, Toward a human blood serum proteome: analysis by multidimensional separation coupled with mass spectrometry, Mol Cell Proteomics 1 (2002), 947-955.

[3] R.C. Bast, Status of tumor markers in ovarian cancer screening, J Clin Oncol 21 (2003), 200s-205s.

[4] L.H. Cazares, B.L. Adam, M.D. Ward, S. Nasim, P.F. Schellhammer, O.J. Semmes and G.L. Wright Jr., Normal, benign, preneoplastic, and malignant prostate cells have distinct protein expression profiles resolved by surface enhanced laser desorption/ionization mass spectrometry, Clin Cancer Res $\mathbf{8}$ (2002), 2541-2552.

[5] H. Christensen, H.H. Thyssen, O. Schebye and A. Berget, Three highly sensitive bedside serum and urine tests for pregnancy compared, Clin Chem 36 (1990), 1686-1688.

[6] T.P. Conrads, M. Zhou, E.F. Petricoin, L. Liotta and T.D. Veenstra, Cancer diagnosis using proteomic patterns, Exp Rev Mol Diag 3 (2003), 411-420.

[7] E.P. Diamandis, Proteomic patterns in serum and identification of ovarian cancer, Lancet 360 (2002), 170.

[8] D.J. Grossklaus, J.A. Smith, S.B. Shappell, C.S. Coffey, S.S. Chang and M.S. Cookson, The free/total prostate-specific antigen ratio (\% fPSA) is the best predictor of tumor involvement in the radical prostatectomy specimen among men with an elevated PSA, Urol Oncol 7 (2002), 195-198.

[9] A.E. Guppy and G.J. Rustin, CA125 response: can it replace the traditional response criteria in ovarian cancer? Oncologist 7 (2002), 437-443.

[10] S. Hanash, Disease proteomics, Nature 422 (2003), 226-232.

[11] J.H. Holland, in: Adaptation in Natural and Artificial Systems: An Introductory Analysis with Applications to Biology, Control, and Artificial Intelligence, J.H. Holland, ed., MIT Press, Cambridge, MA USA.

[12] H.J. Issaq, T.P. Conrads, D.A. Prieto, R. Tirumalai and T.D. Veenstra, SELDI-TOF MS for diagnostic proteomics, Anal Chem 75 (2003), 149A-155A.

[13] K.K. Jain, Role of proteomics in diagnosis of cancer, Technol Cancer Res Treat 1 (2002), 281-286.

[14] C. Kainz, Early detection and preoperative diagnosis of ovarian carcinoma, Wien. Med. Wochenschr 146 (1996), 2-7.

[15] Y. Kohonen, Biological Cybernetics 43 (1982), 59-69.

[16] Y. Kohonen, Proc. Inst. Electrical Electronics Eng. 78 (1990), 1464-1480.

[17] J. Li, Z. Zhang, J. Rosenzweig, Y.Y. Wang and D.W. Chan, Proteomics and bioinformatics approaches for identification of serum biomarkers to detect breast cancer, Clin Chem 48 (2002), 1296-1304.

[18] R.F. Ozols, S.C. Rubin, G.M. Thomas, and S.J. Robboy, in: Epithelial Ovarian Cancer, W.J. Hoskins, C.A. Perez and R.C. Young, eds, Principles and Practice of Gynecologic OncologyLippincott Williams and Wilkins, Philadelphia, PA USA, pp. 981-1058.

[19] E.F. Petricoin, A.M. Ardekani, B.A. Hitt, P.J. Levine, V.A. Fusaro, S.M. Steinberg, G.B. Mills, C. Simone, D.A. Fishman, 
E.C. Kohn and L.A. Liotta, Use of proteomic patterns in serum to identify ovarian cancer, Lancet 359 (2002), 572-577.

[20] E.F. Petricoin, D.K. Ornstein, C.P. Paweletz, A. Ardekani, P.S. Hackett, B.A. Hitt, A. Velassco, C. Trucco, L. Wiegand, K. Wood, C.B. Simone, P.J. Levine, W.M. Linehan, M.R. Emmert-Buck, S.M. Steinberg, E.C. Kohn and L.A. Liotta, Serum proteomic patterns for detection of prostate cancer, $J$. Natl. Cancer Inst 94 (2002), 1576-1578.

[21] Y. Qu, B.L. Adam, Y. Yasui, M.D. Ward, L.H. Cazares, P.F. Schellhammer, Z. Feng, O.J. Semmes and C.L. Wright Jr., Boosted decision tree analysis of surface-enhanced laser desorption/ionization mass spectral serum profiles discriminates prostate cancer from noncancer patients, Clin Chem $\mathbf{4 8}$ (2002), 1835-1843.

[22] R. Tirumalai, K.C. Chan, D.A. Prieto, H.J. Issaq, T.P. Conrads and T.D. Veenstra, Characterization of the low molecular weight human serum proteome, Mol. Cell. Proteomics 2 (2003), 1096-1103.
[23] S.L. Wu, H. Amato, R. Biringer, G. Choudhary, P. Shieh and W.S. Hancock, Targeted proteomics of low-level proteins in human plasma by LC/MSn: using human growth hormone as a model system, J. Proteome Res 1 (2002), 459-465.

[24] K. Yanagisawa, Y. Shyr, B.J. Xu, P.P. Massion, P.H. Larsen, B.C. White, J.R. Roberts, M. Edgerton, A. Gonzalez, S. Nadaf, J.H. Moore, R.M. Caprioli and D.P. Carbone, Proteomic patterns of tumour subsets in non-small-cell lung cancer, Lancet 362 (2003), 433-439.

[25] L.R. Yu, M.D. Johnson, T.P. Conrads, R. D. Smith, R.S. Morrison and T.D. Veenstra, Proteome analysis of camptothecintreated cortical neurons using isotope-coded affinity tags, Electrophoresis 23 (2002), 1591-1598.

[26] Z. Xiao, T.P. Conrads, D.A. Lucas, G.M. Janini, C.F. Schaefer, K.H. Buetow, H.J. Issaq and T.D. Veenstra, Direct ampholytefree liquid phase isoelectric peptide focusing: Application to the human serum proteome, Electrophoresis, In press. 


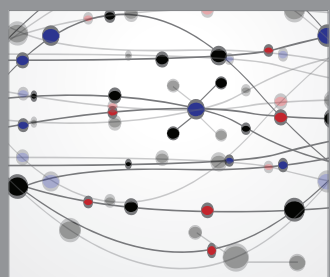

The Scientific World Journal
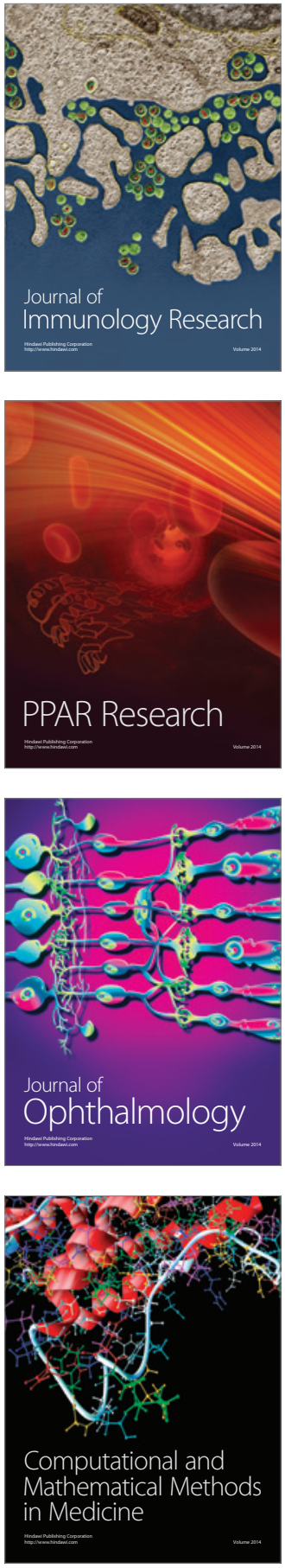

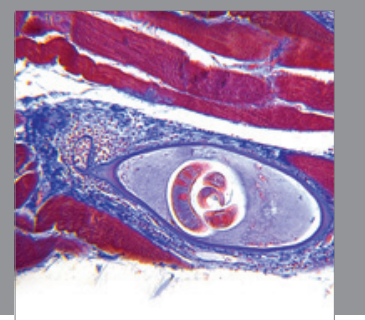

Gastroenterology

Research and Practice
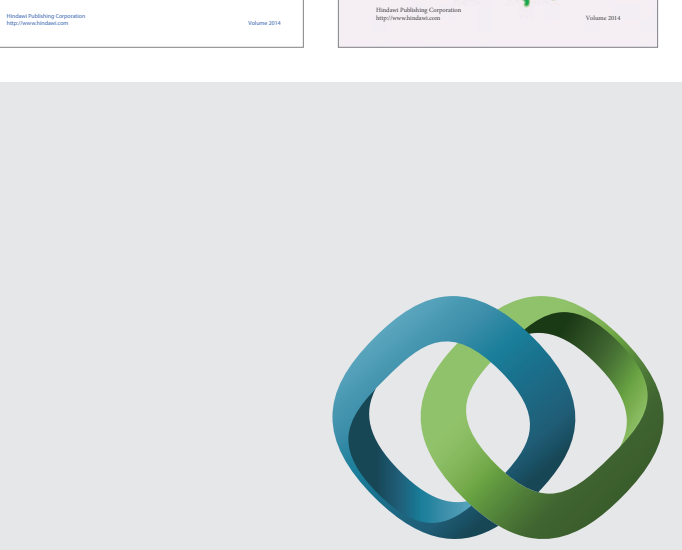

\section{Hindawi}

Submit your manuscripts at

http://www.hindawi.com
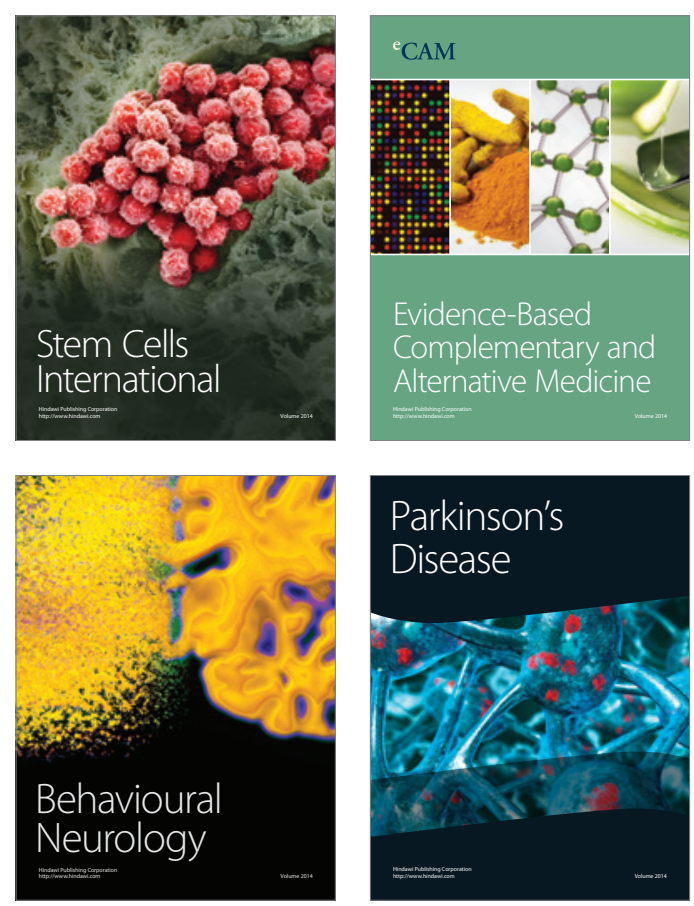

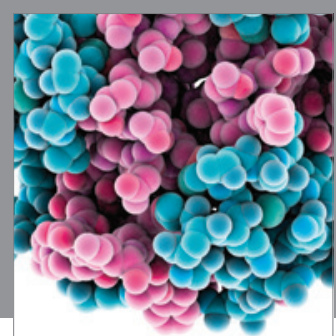

Journal of
Diabetes Research

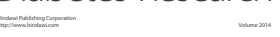

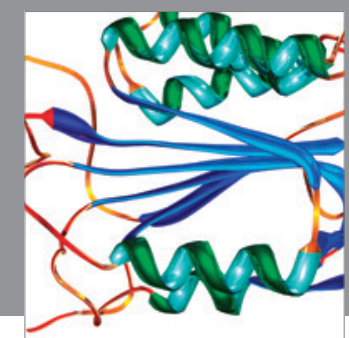

Disease Markers
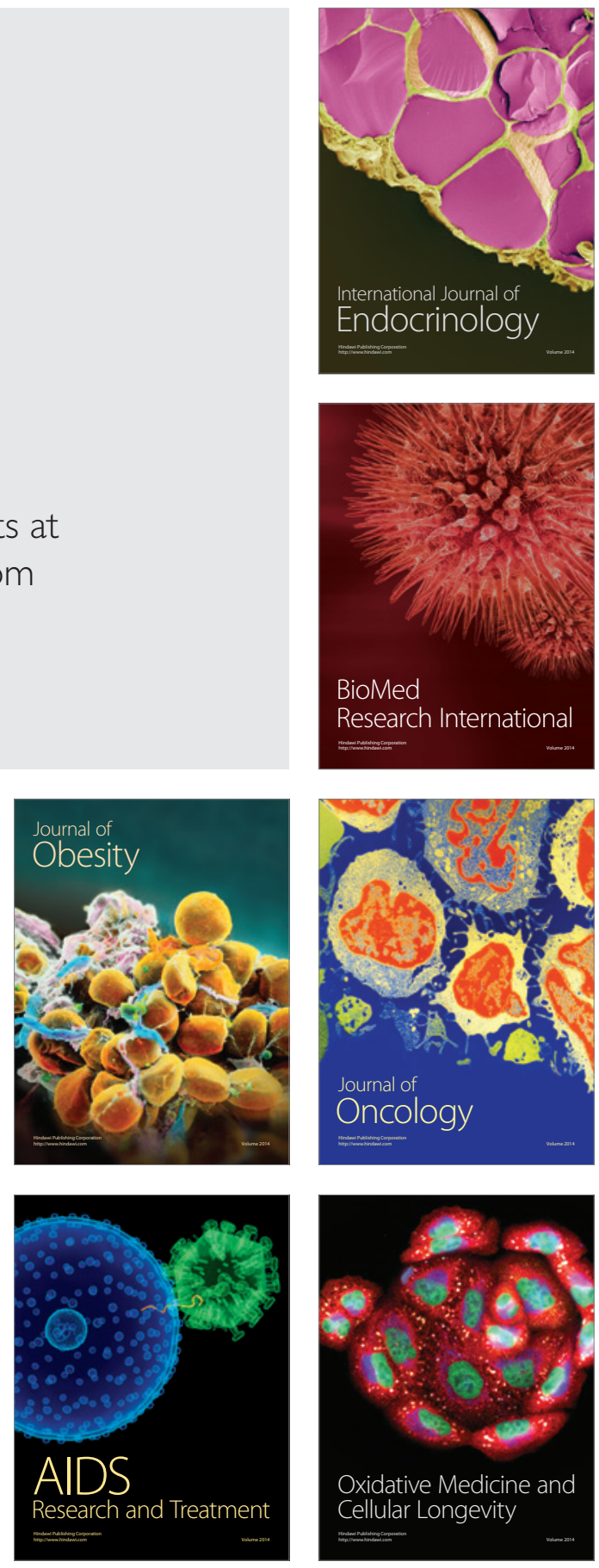